\title{
Joule effect self-heating of epoxy composites reinforced with graphitic nanofillers
}

\author{
S. G. Prolongo ${ }^{1} \cdot$ R. Moriche $^{1} \cdot$ G. Del Rosario ${ }^{2} \cdot$ A. Jiménez-Suárez $^{1}$. \\ M. G. Prolongo ${ }^{3} \cdot$ A. Ureña ${ }^{1}$
}

\begin{abstract}
Self-heating of conductive nanofilled resins due to the Joule effect is interesting for numerous applications, including computing, self-reparation, self-post-curing treatment of resins, fabrication of adhesive joints, de-icing coatings and so on. In this work, we study the effect of the nature and amount of graphitic nanofiller on the self-heating of epoxy composites.

The addition of graphitic nanofillers induced an increase in the thermal conductivity of the epoxy resins, directly proportional to the nanofiller content. Percolation was not observed because of the heat transport through phonons. In contrast, the electrical conductivity curves present a clear percolation threshold, due to the necessity of an electrical percolation network. The electrical threshold is much lower for composites reinforced with carbon nanotubes (CNTs, $0.1 \mathrm{wt} . \%)$ than for the resin filled with graphene nanoplatelets (GNPs, $5 \%$ ). This fact is due to their very different specific areas.

The composites filled with CNTs reach higher temperatures than the ones reinforced with GNPs, applying low electrical voltage because of their higher electrical conductivity. In contrast, the self-heating is more homogeneous for the GNP/epoxy resins due to their higher thermal conductivity. It was also confirmed that the self-heating is repetitive in several cycles, reaching the same temperature when the same voltage is applied.
\end{abstract}

S. G. Prolongo

silvia.gonzalez@urjc.es

1 Department of Materials Science and Engineering, Universidad Rey Juan Carlos, (Madrid), 28933 Móstoles, Spain

2 Technological Support Center, Universidad Rey Juan Carlos, (Madrid), 28933 Móstoles, Spain

3 Department of Aerospace Materials and Production, E.T.S.I. Aeronáutica y del Espacio, Universidad Politécnica de Madrid, 28040 Madrid, Spain
Keywords Self-heating $\cdot$ Joule effect $\cdot$ Composite $\cdot$ Carbon nanotube $\cdot$ Graphene

\section{Introduction}

For two decades, significant research has been carried out regarding the addition of carbon nanotubes (CNTs) into epoxy matrices in order to enhance the electrical, mechanical and thermal properties [1, 2]. Recently, graphene nanoplatelets (GNPs) have become an interesting candidate as a nanofiller for these polymer matrices [3-6]. Both these graphitic nanofillers possess excellent electrical and thermal conductivities, along with very high moduli. However, these properties strongly depend on the geometry, size and aspect ratio of the fillers together with the obtained morphology of the composite (dispersion degree, exfoliation, orientation and so on).

Nanoreinforced composites have already shown promising results for creating electrically conductive polymers. Electrical current induces the Joule heating effect, or self-heating, into nanoreinforced polymers [7-9]. This heating can be used for different applications. One example consists of burning out conductive CNTs and leaving semi-conductive CNTs for computer applications [7, 8]. Another application is the self-reparation of composites by self-post-curing treatment of reinforced resins or adhesives by the application of electrical current [8]. Selfheating is also being researched for de-icing coatings [10].

Heating by the Joule effect depends on both the electrical and thermal conductivities of the composite, which are strongly influenced by the nanofiller properties. The thermal conductivities of different graphitic fillers are in the range of $\sim 1 \mathrm{~W} / \mathrm{mK}$ for amorphous carbon to $\sim 5000 \mathrm{~W} / \mathrm{mK}$ for monolayer graphene [11]. The thermal conductivity of multi-walled CNTs is close to $3000 \mathrm{~W} / \mathrm{mK}$ at room temperature [11]. In contrast, graphene presents very high in-plane $(>5000 \mathrm{~W} /$ 
$\mathrm{mK}$ ) and relatively low out-of-plane thermal conductivities $[12,13]$. The electrical conductivity of both these materials is very high, reaching values of $\sim 10,000 \mathrm{~S} / \mathrm{m}$ in the main plane of graphene and the main CNT axis [14].

In this work, we study the effect of self-heating caused by the transportation of electrical current in epoxy composites filled with GNPs and CNTs. For this purpose, the thermal and electrical behavior of these composites is firstly analyzed. The influence of the nanofiller content added is also analyzed.

\section{Experimental}

\section{Sample preparation}

Graphene was supplied by XG Science, under the trade name of M25. Its purity was $99.5 \%$ by weight and consisted of nanoparticles with an average thickness of $6-8 \mathrm{~nm}$ and an average lateral size of $25 \mu \mathrm{m}$. CNTs were supplied by Nanocyl with the commercial name NC3150. They had an average diameter of $9.5 \mathrm{~nm}$ and were $1 \mu \mathrm{m}$ in length. These data, provided by the manufacturer, match the characterization performed in previous works $[15,16]$. Epoxy resin was obtained from a basic diglycidilether bisphenol A (DGBEA) monomer (Araldite LY556) cured with an aromatic amine (Araldite XB3473), both purchased from Huntsman.

The dispersion procedure used was based on a method previously published $[15,16]$. The method is based on several cycles of calendaring with different rollers gaps, from 15 to $5 \mu \mathrm{m}$, and an increasing velocity per cycle of 250,300 and $350 \mathrm{rpm}$. The dispersion procedure was carried out in the monomer of the resin. Once the dispersion was completed, the mixture was degassed under vacuum $(40 \mathrm{mbar})$ at $80^{\circ} \mathrm{C}$ for $15 \mathrm{~min}$. After the elimination of the occluded gases, hardener was added in a 100:23 (LY556:XB3473) weight ratio to achieve the stoichiometric proportion. The mixture was then cured at $140^{\circ} \mathrm{C}$ for $8 \mathrm{~h}$ in an open mold.

The optimum content of nanofillers was obtained as a function of the percolation threshold of each studied system, as previously determined $[15,16]$. The studied CNT/epoxy system presents an electrical threshold lower than $0.1 \mathrm{wt} . \%$, while the percolation is reached at values higher than $1 \mathrm{wt} \%$ for the GNP/epoxy composites. Due to this fact, the CNT contents added were $0.1,0.25$ and $0.5 \mathrm{wt} . \%$, while the GNP contents were $1.5,2,3,5$ and $8 \mathrm{wt} \%$.

\section{Characterization}

\section{Dynamic mechanical thermal analysis (DMTA)}

Dynamic mechanical thermal analysis (DMTA) of the cured samples was performed in dual cantilever bending mode using a DMTA V Rheometric Scientific instiument. Measurements were done at $1 \mathrm{~Hz}$ with the temperature increasing from 30 to $220^{\circ} \mathrm{C}$ at a heating rate of $2{ }^{\circ} \mathrm{C} \cdot \mathrm{min}^{-1}$. Specimens dimensions were $35 \times 12 \times 1.5 \mathrm{~mm}^{3}$. The elastic or storage modulus $(E)$, loss modulus $\left(E^{\prime \prime}\right)$ and loss tangent $(\tan \delta)$ were recorded as a function of temperature. The maxima in the $\tan \delta$-temperature curves were determined to identify the $\alpha$-relaxations associated with the glass transitions.

\section{Thermal conductivity measurements}

The thermal diffusivity was measured with Laserflash LFA 457 MicroFlash equipment, applying a temperature scanning from 20 to $200^{\circ} \mathrm{C}$. In heat transfer analysis, thermal diffusivity is the thermal conductivity divided by the density and specific heat capacity at constant pressure. It measures the ability of a material to conduct thermal energy relative to its ability to store thermal energy.

In order to calculate the thermal conductivity, the specific heat capacity was determined by differential scanning calorimetry (DSC, Mettler Toledo mod.822e), while the density of the composites at room temperature was measured by a Mettler Toledo balance $( \pm 0.001 \mathrm{mg})$, equipped with a density measurement kit.

\section{Electrical conductivity measurements}

The electrical conductivity was measured following the standard ASTM D257. A SourceMeter instrument (Keithley 2410, Keithley Instruments) connected through an interface GPIB to a PC was used. The electrical resistance was determined by calculating the slope current-voltage characteristic curve, from which the electrical conductivity can be determined taking into account the geometry of the specimens $\left(10 \times 10 \times 1 \mathrm{~mm}^{3}\right)$.

\section{Determination of the joule effect}

The self-heating caused by the transportation of electrical current was determined using the SourceMeter instrument (Keithley 2410) together with a laser IR digital thermometer. Different experiments were designed in order to analyze diverse parameters, such as the temperature increase as a function of the electrical current and the reproducibility of studied systems, for applications such as electrical sensors, positive temperature coefficient thermistors and deicing systems. Finally, the study of self-heating homogeneity was carried out by thermography (FLIR E50).

\section{Results and discussion}

\section{Determination of thermal behavior}

Considering that the application of the studied materials would imply their use at relatively high temperatures, it is very 
important to determine the maximum working temperature of these systems. Taking into account that these composites are based on thermosetting resins, it is possible to associate this temperature to the glass transition temperature. Figure 1 and Table 1 show the DMTA results. As expected [1, 3], the nanofilled epoxy resins present relatively high moduli at room temperature, which slightly decrease when the temperature increases. An abrupt drop in rigidity occurs at relatively high temperature, associated with the $\alpha$-relaxation from the storage state to the rubber of the thermosetting polymer. The addition of graphitic nanofillers induces an increase in storage moduli at room temperature but, in addition, a decrease in the glass transition temperatures of the systems. The highest modulus measured, which corresponds to the epoxy resin doped with $8 \mathrm{wt} . \%$ GNPs, is $42 \%$ higher than that of the neat epoxy resin. However, the incorporation of higher GNP percentages does not necessarily mean a higher rigidity of material because dispersion problems tend to appear. In fact, this is the main problem in the CNT/epoxy system. CNT addition scarcely induces a $15 \%$ increase in the modulus, regardless of CNT content. This fact could be associated with the presence of agglomerations due to the very high specific surface area of nanotubes.

The $\alpha$-relaxation temperature of the epoxy resin increases slightly by $1-3{ }^{\circ} \mathrm{C}$ with the addition of CNTs and GNPs, probably associated with the mobility impairment of the polymeric segments. However, the addition of upper contents of nanofillers with high specific surface areas induces a decrease in the glass transition temperature due to dispersion problems, as occurs for the resin doped with $0.5 \mathrm{wt} . \%$ CNTs.

\section{Thermal and electrical conductivity measurements}

Figure 2 shows the variation of thermal conductivity due to the addition of different contents of graphitic nanofillers. The
Fig. 1 DMTA results for the variation of storage modulus, loss modulus and loss tangent as a function of temperature for epoxy resins reinforced with CNTs (a) and GNPs (b)
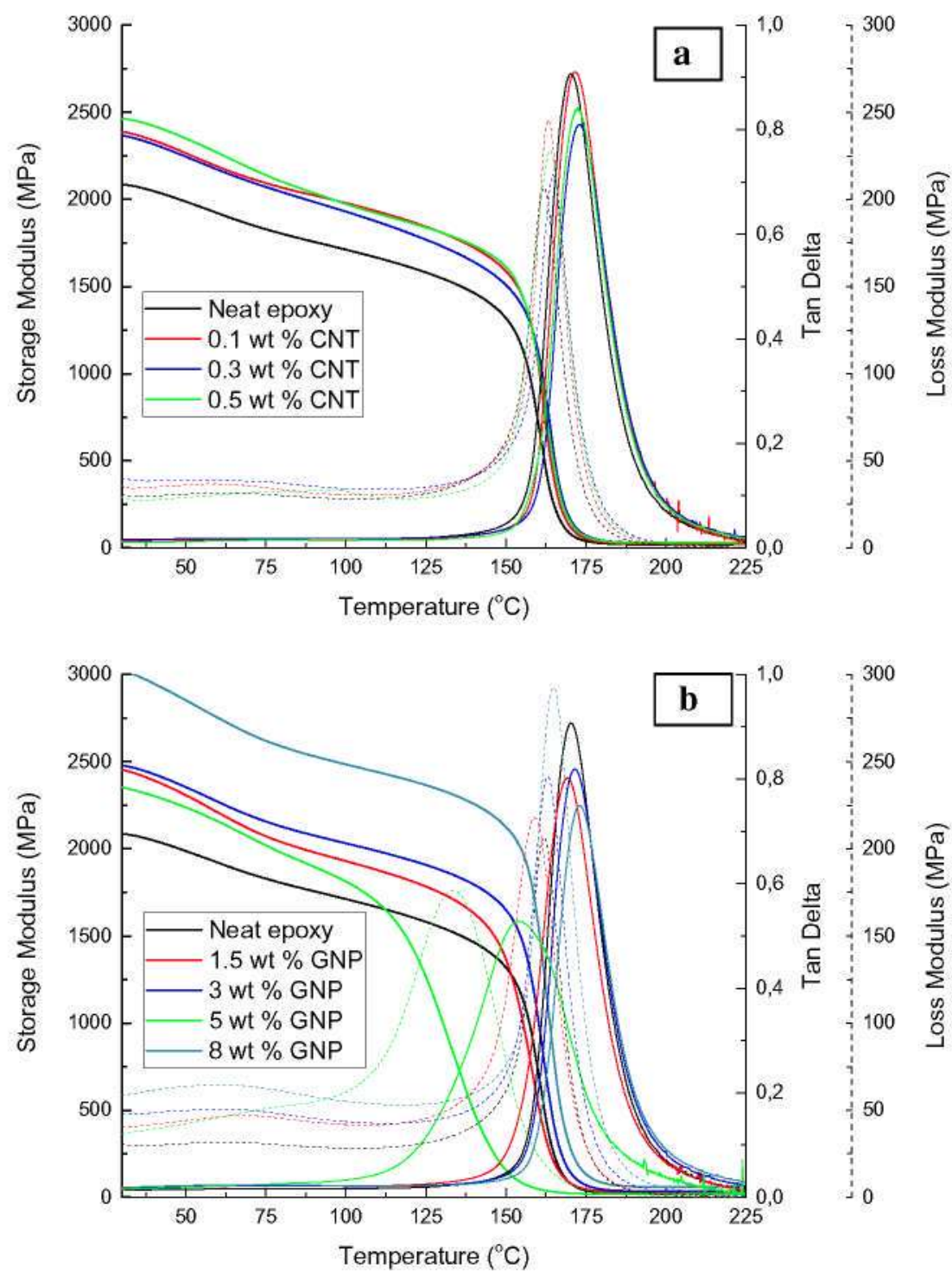
Table 1 DMTA results for the values of storage modulus ( $\left.E^{\prime}\right)$ at room temperature and $\alpha$-relaxation temperature $\left(\mathrm{T}_{\mathrm{g}}\right)$ for epoxy resins reinfored with different contents of CNTs and GNPs

\begin{tabular}{lll}
\hline Sample & $\mathrm{E}^{\prime}(\mathrm{GPa})$ at $30^{\circ} \mathrm{C}$ & $\mathrm{T}_{\mathrm{g}}\left({ }^{\circ} \mathrm{C}\right)$ \\
\hline Resin & $2.13 \pm 0.06$ & $170.4 \pm 0.1$ \\
$0.1 \% \mathrm{CNT}$ & $2.41 \pm 0.07$ & $171.8 \pm 0.1$ \\
$0.3 \% \mathrm{CNT}$ & $2.06 \pm 0.20$ & $166.0 \pm 0.2$ \\
$0.5 \% \mathrm{CNT}$ & $2.38 \pm 0.12$ & $172.3 \pm 0.1$ \\
$1.5 \% \mathrm{GNP}$ & $2.30 \pm 0.20$ & $169.8 \pm 0.7$ \\
$2.0 \% \mathrm{GNP}$ & $1.81 \pm 0.09$ & $172.5 \pm 0.3$ \\
$3.0 \% \mathrm{GNP}$ & $2.21 \pm 0.35$ & $171.5 \pm 0.2$ \\
$5.0 \% \mathrm{GNP}$ & $2.30 \pm 0.10$ & $172.1 \pm 0.4$ \\
$8.0 \% \mathrm{GNP}$ & $3.05 \pm 0.10$ & $171.3 \pm 2.5$ \\
\hline
\end{tabular}

thermal conductivity increases proportionally with the content of nanofillers added, when GNPs are used. The thermal conductivity of the epoxy composite filled with 8 wt.\% GNPs is three times higher than that of the pristine matrix. No clear percolation threshold was found. This behavior has been already observed by other authors $[4,17]$. The thermal conductivity is based on phonon transport and therefore it depends on both the filler and matrix, which contribute to the heat flow. In this case, the thermal conductivities of the filler $(\sim 3000 \mathrm{~W} /$ $\mathrm{mK})$ and matrix $(\sim 0.1 \mathrm{~W} / \mathrm{mK})$ are very different. Because of this, the presence of large interfacial thermal resistance leads to the lack of a percolation curve in the thermal conductivity [4]. The addition of CNTs may cause interruption of phonon transport trough graphene layers when they are positioned surrounding the GNPs thus increasing the number of contact interfaces between nanoparticles and, consequently, they do not cause a positive effect on the thermal conductivity.

In contrast with the thermal conductivity, the curves of electrical conductivity show a percolation threshold as a

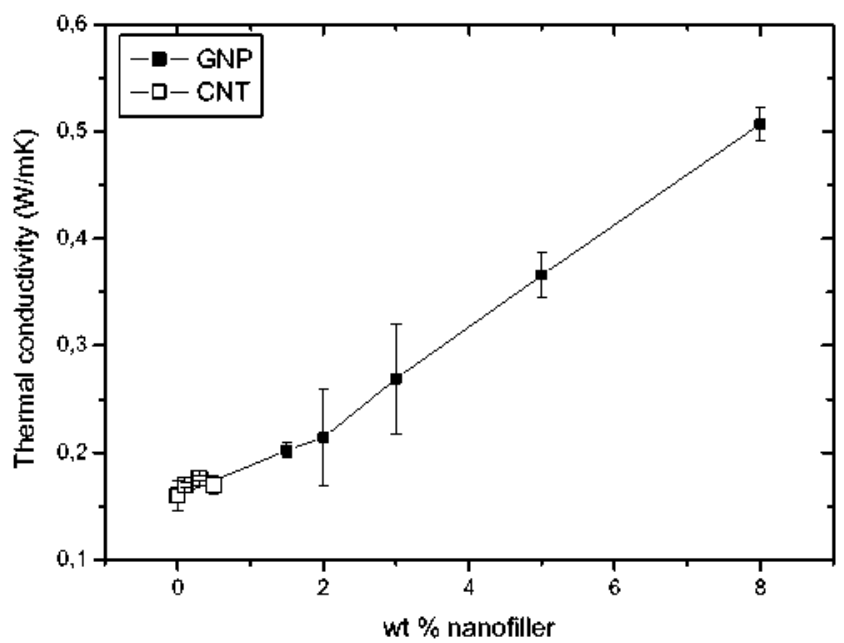

Fig. 2 Thernal conductivity of epoxy composites reinforced with CNTs and GNPs function of nanofiller content (Fig. 3). This means that for nanofiller percentages lower than the percolation threshold, the composite is an insulator but its electrical conductivity increases abruptly at higher contents. The electrical percolation is due to the electrical conductivity and only depends on the connectivity between conductive nanofillers. This means that the formation of an interconnecting network is necessary to obtain electrically conductive materials. For this reason and, as expected, the percolation threshold of the CNT reinforced composites is much lower $(0.1 \mathrm{wt} . \%)$ than the ones filled with GNPs (close to 8 wt. \%). This behavior is explained by the much higher specific area of the nanotubes $(>1000 \mathrm{~g} /$ $\mathrm{m}^{2}$ ) than the GNPs (in the range of $100-300 \mathrm{~g} / \mathrm{m}^{2}$ ) and the more efficient $2 \mathrm{D}$ geometry to form an effective network [3]. In addition, the electrical conductivity of the CNT/epoxy composites, which reaches a value of $0.2 \mathrm{~S} / \mathrm{m}$, is considerably higher than the conductivity of the epoxy resins reinforced with GNPs, in the range of $0.004 \mathrm{~S} / \mathrm{m}$. This fact can be associated with the more efficient geometry of CNTs in forming an electrical network and the higher electrical conductivity of these nanofillers.

\section{Self-heating by the joule effect}

The first test to characterize the Joule effect on the studied composites consisted of the determination of the temperature reached as a function of the applied voltage. Figure 4 shows the current-voltage curves of the studied materials, along with the increment of temperature produced by the Joule effect. In all cases, the current increases linearly with the applied voltage, indicating that all studied materials follow Ohm's law. The obtained results show that for the same type of nanofiller, the increment of nanofiller content induces an augment in the electrical conductivity, which is observed by an increase of electrical current for the same voltage applied. Additionally, this increase in the electrical current also implies a higher

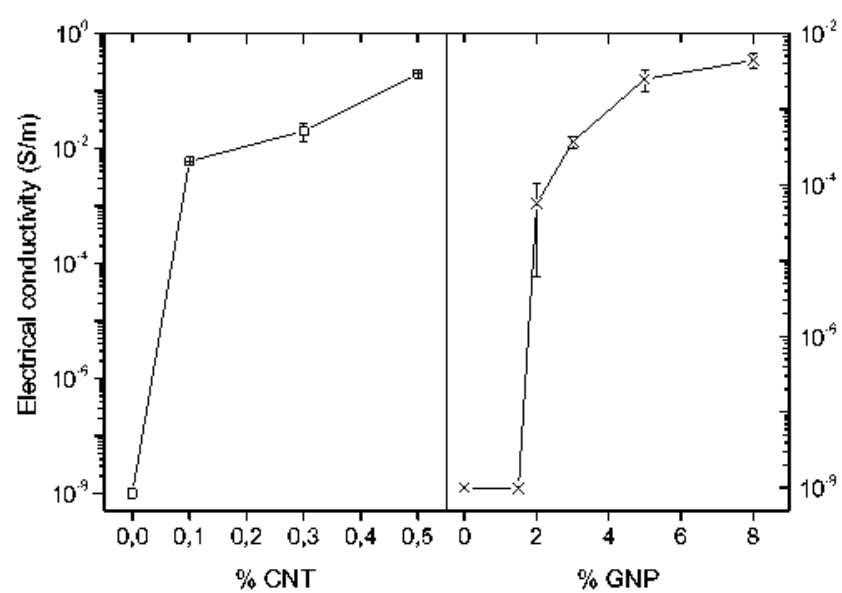

Fig. 3 Electrical conductivity of epoxy composites reinforced with C.NTs and GNPs 
Fig. 4 Electrical current (solid line) and increment of temperature (dot lines) associated as a function of applied voltage for epoxy composites reinforced with CNTs (a) and GNPs (b)
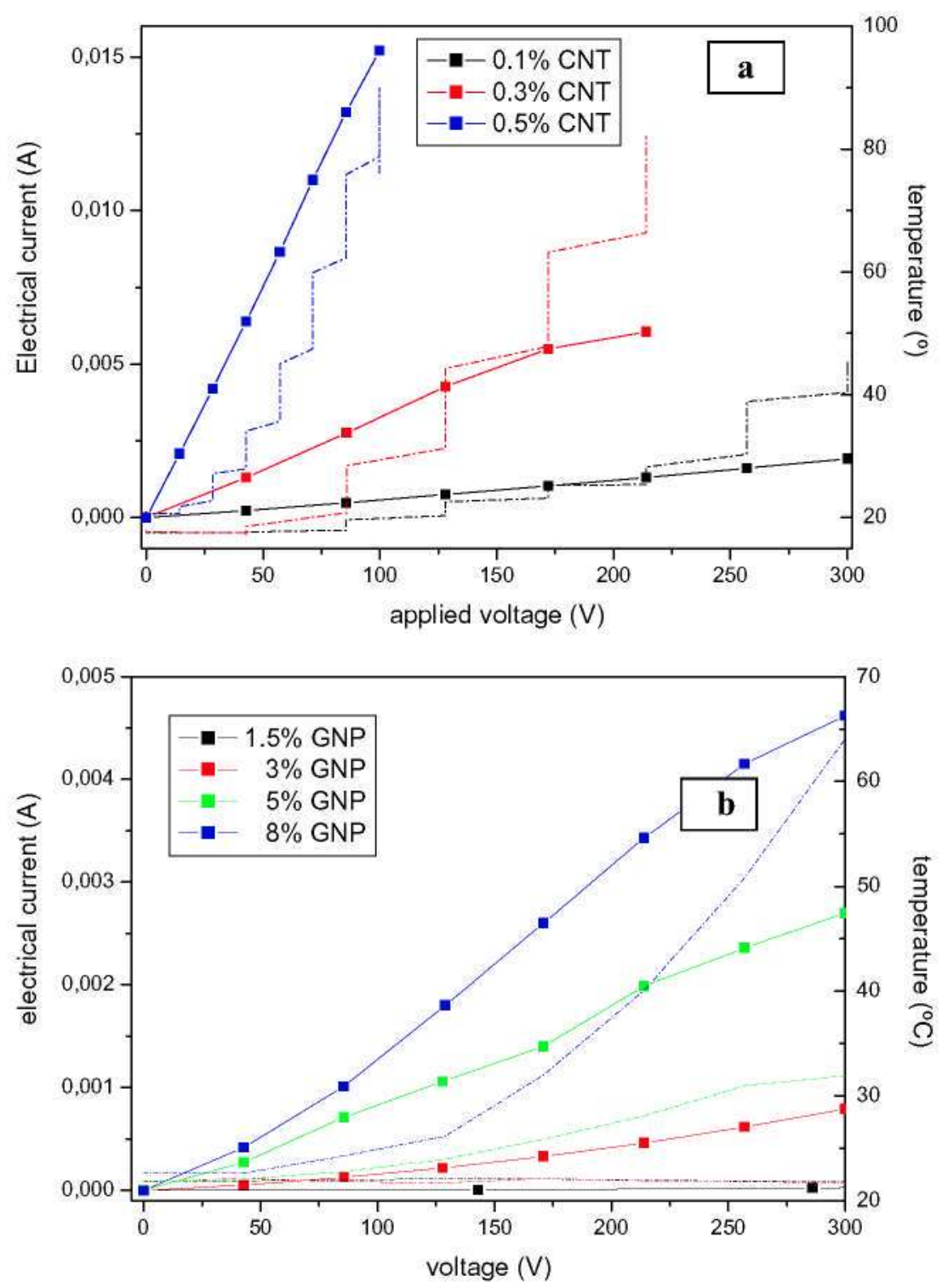

increment of temperature due to the Joule effect. This is explained because the studied systems behave as semiconductor materials, since their electrical conductivity is relatively low, in the range of $10^{-3}-10^{-1} \mathrm{~S} / \mathrm{m}$.

It is noteworthy that the self-heating capability strongly depends on the graphitic nanofiller incorporated into the polymer matrix. This assentation is based on the fact that for the same electrical current, the temperature reached is different for both studied systems, epoxy resins reinforced with CNTs and GNPs. This means that the self-heating caused by the Joule effect is not only influenced by the electrical resistance of the material, but it is also dependent on the material itself. Figure 5 shows a summary of the electrical conductivity of studied materials and the maximum temperature reached as a function of the applied voltage. It is appreciated that composites with higher electrical conductivities reach higher temperatures at lower applied voltages.
In order to analyze the homogeneity of the selfheating of these materials, the composites with the highest contents of CNTs and GNPs were selected to study them by thermography. Taking into account the results shown in Fig. 4, both are able to reach relative high temperatures, close to $75{ }^{\circ} \mathrm{C}$, but the applied voltages are different, $75 \mathrm{~V}$ for the epoxy composite with 0.25 wt. $\%$ CNTs and $200 \mathrm{~V}$ for the resin reinforced with $8 \%$ GNPs. The experiment consisted of applying these selected voltages, 75 and $300 \mathrm{~V}$, in order to observe the homogeneity of temperature on the material. Figure 6 shows the thermographic images. It can be clearly appreciated that the reached temperature is similar, $70-80{ }^{\circ} \mathrm{C}$, for both materials, but the homogeneity of the selfheating is higher for the composite reinforced with GNPs. In fact, the composite filled with 0.25 wt.\% CNTs presents areas with different colors, meaning that 


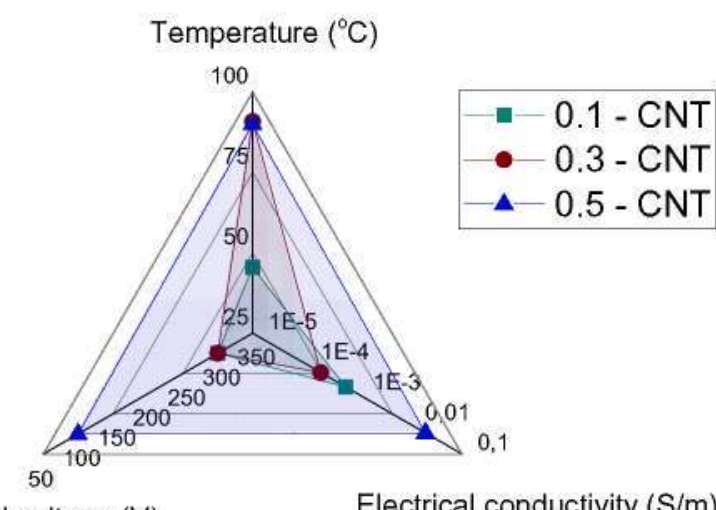

Applied voltage (V)

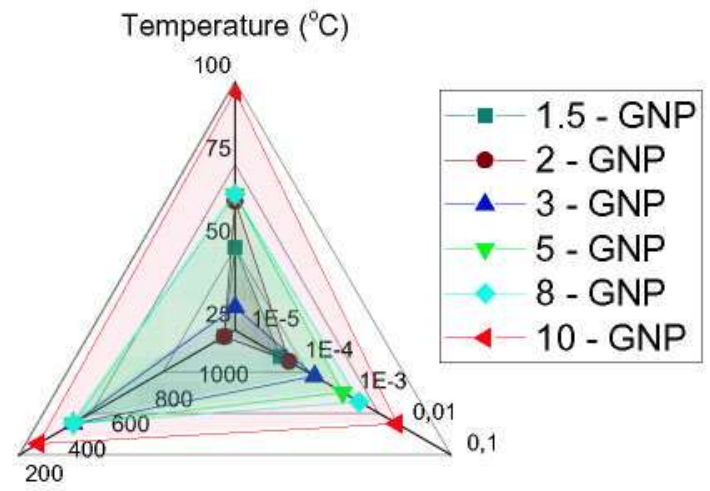

Applied voltage (V)

Electrical conductivity (S/m)

Fig. 5 Electrical conductivity of the epoxy composites reinforced with CNTs and GNPs, together with the temperature reached as a function of applied voltage

the temperature is different, ranging from 60 to $80^{\circ} \mathrm{C}$. In contrast, the material reinforced with $8 \%$ GNPs shows a homogeneous temperature over its surface. This is associated with the different thermal conductivity of the studied composites. It is well known that the heating due to the Joule effect is mainly produced in the electrical contacts. In the case of composites reinforced with GNPs, the heating of the sample is homogeneous due to its relatively high thermal conductivity. The low thermal conductivity of composites with CNTs induces the appearance of regions with different temperatures.

Finally, in terms of industrial applications, it is necessary to analyze the reproducibility of the self-heating caused by the transport of electrical current. Figure 7 shows some of the cyclic curves obtained by applying the same voltage, several times, during a relative short period of time. The experiment consists of periodic cycles of $50 \mathrm{~V}$ for $30 \mathrm{~min}$, and then removal of the current for $5 \mathrm{~min}$. It is clearly observed that the self-heating is totally reproducible and there is no hysteresis effect. In all cycles, the maximum temperature reached is the same because the electrical current transported is only a function of the applied voltage.
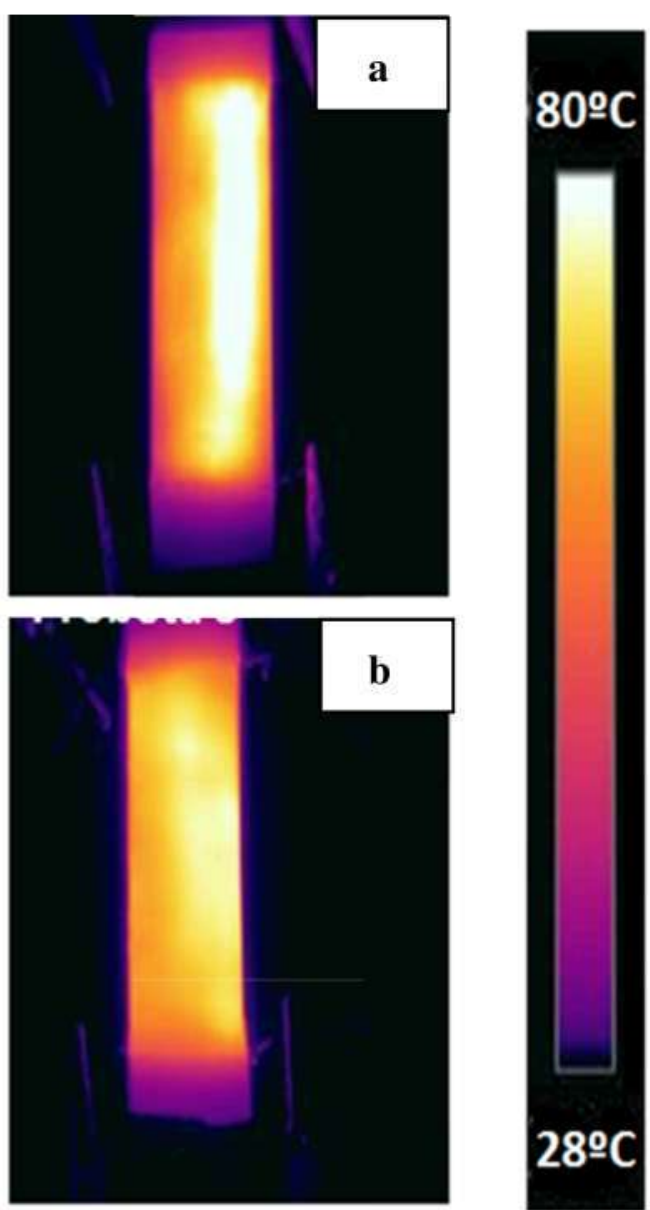

Fig. 6 Thermographic images of composites reinforced with 0.25 wt. \% CNTs (a) and 8 wt.\% GNPs (b) when a voltage of 75 and $300 \mathrm{~V}$ is applied, respectively

\section{Conclusions}

Self-heating by the Joule effect was studied in composites reinforced with CNTs and GNPs. Firstly, it was probed that the addition of graphitic nanofillers into epoxy resin scarcely affects the glass transition temperature. In contrast, these nanofillers induce an important increase in the glassy storage modulus.

Alternatively, we confirm that the nanofiller addition contributes with different effects on electrical and thermal conductivity of the resin. Graphitic nanofillers induces an increase of the thermal conductivity of the epoxy resin, directly proportional to the nanofiller content. Percolation was not observed because of the heat transport through phonons. In contrast, the electrical conductivity curves present a clear percolation threshold, due to the necessity of an electrical percolation network. The electrical threshold of CNT reinforced composites is much lower than those filled with GNPs, due to their very different specific areas and geometries. Both aspects conferred by the two types of nanofillers are needed, thus making interesting the use of combination of the two of them. Electrical conductivity is needed to create the Joule Effect 


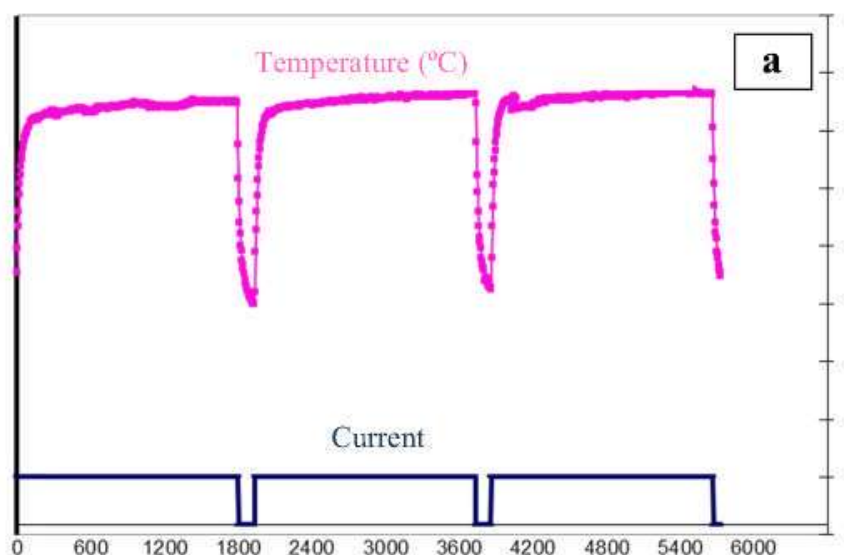

Time (s)

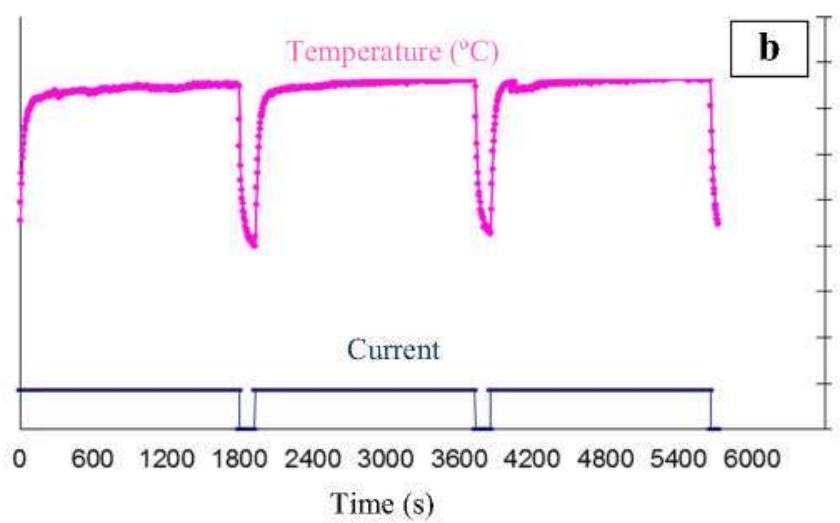

Fig. 7 Cyclic test of measurement of self-heating by the Joule effect, applying $50 \mathrm{~V}$ for $30 \mathrm{~min}$ and epoxy composites reinforced with $0.5 \mathrm{wt} \% \mathrm{CNTs}$ and $10 \mathrm{wt} \% \mathrm{GNPs}$

while thermal conductivity allows homogenization of heat generated, particularly interesting, as the nanocomposites are not completely homogeneous which could lead to heat concentration in certain material regions.

In this work, we analyze the self-heating of the nanofilled composites by the Joule effect. We confirm that the heating of CNT composites is more effective, reaching higher temperatures, than the ones reinforced with GNPs, applying low electrical voltage. The reason is the higher electrical conductivity of the CNT/epoxy composites. In contrast, the self-heating is more homogeneous on GNP/epoxy resins due to their higher thermal conductivity. Finally, we have confirmed that the self-heating of these materials is repetitive in several cycles, reaching the same temperature when the same voltage is applied.

Acknowledgments The authors would like to thank the Ministerio de Economia y Competitividad of the Spanish government (Project MAT2013-46695-C3-1-R) and Comunidad de Madrid (Project P2013/ MIT-2862)

\section{References}

1. Spitalskya Z, Tasisb D, Papagelisb K, Galiotisa C (2010) Carbon nanotube-polymer composites: chemistry, processing, mechanical and electrical properties. Prog Polym Sci 35: $357-401$

2. Liu Y, Kumar S (2014) Polymer/carbon nanotube nano composite fibers - a review. ACS Appl Mater Inter 6:6069-6087

3. Mittala G, Dhanda V, Rheea KY, Parkb SY, Leec WR (2015) A review on carbon nanotubes and graphene as fillers in reinforced polymer nanocomposites. J Ind Eng Chem 21:11-25

4. Chandrasekaran S, Seidel C, Schulte K (2013) Preparation and characterization of graphite nano-platelet (GNP)/epoxy nanocomposite: mechanical, electrical and thermal properties. Euro Polym J 49:3878-3888

5. Sun X, Sun H, Li H, Peng H (2013) Developing polymer composite materials: carbon nanotubes or graphene. Adv Mater 25:5153-5176

6. Saravanan N, Rajasekar R, Mahalakshmi S, Sathishkumar TP, Sasikumar KSK, Sahoo S (2014) Graphene and modified graphene-based polymer nanocomposites - a review. J Reinf Plast Compos 33:1158-1170

7. Chien AT, Cho S, Joshi Y, Kumar S (2014) Electrical conductivity and joule heating of polyacrylonitrile/carbon nanotube composite fibers. Polymer 55:6896-6905

8. Mas B, Fernandez-Blazquez JP, Duval J, Bunyan H, Vilatela JJ (2013) Thermoset curing through joule heating of nanocarbons for composite manufacture, repair and soldering. Carbon 63:523-529

9. Zeng Y, Lu G, Wang H, Du J, Ying Z, Liu C (2014) Positive temperature coefficient thermistors based on carbon nanotube/ polymer composites. Sci Rep 4:-6684

10. Chua H, Zhanga Z, Liub Y, Leng J (2014) Self-heating fiber reinforced polymer composite using meso/macropore carbon nanotube paper and its application in deicing. Carbon 66:154-163

11. Pop E, Mann D, Wang Q, Goodson K, Dai HJ (2006) Thermal conductance of an individual single-wall carbon nanotube above room temperature. Nano Lett 6:96-100

12. Pop E, Varshney V, Roy AK (2012) Thermal properties of graphene: fundamentals and applications. MRS Bull 37:-1273

13. Ghosh S, Calizo I, Teweldebrhan D, Pokatilov EP, Nika DL, Balandin AA, Bao W, Miao F, Lau CN (2008) Extremely high thermal conductivity of graphene: prospects for thermal management applications in nanoelectronic circuits. Appl Phys Lett 92:151911

14. Marinhoa B, Ghislandia M, Tkalyac E, Koningc CE, Witha G (2012) Electrical conductivity of compacts of graphene, multiwall carbon nanotubes, carbon black, and graphite powder. Powder Technol 221:351-358

15. Prolongo SG, Burón M, Gude MR, Chaos-Morán R, Campo M, Ureña A (2008) Effects of dispersion techniques of carbon nanofibers on the thermo-physical properties of epoxy nanocomposites. Compos Sci Technol 68:2722-2730

16. Prolongo SG, Jiménez-Suárez A, Moriche R, Ureña A (2013) In situ processing of epoxy composites reinforced with grapheme nanoplatelets. Compos Sci Technol 86:185-191

17. Gu J, Yang $X$, Lv Z, Liang $C$, Zhang Q (2015) Functionalized graphite nanoplatelets/epoxy resin nanocomposites with high thermal conductivity. Int J Heat Mass Transf 92:15-22 